\title{
Codes and shifted codes of partitions
}

\author{
J. T. Hird \\ Department of Mathematics \\ North Carolina State University, North Carolina, USA \\ jthird@ncsu.edu \\ Naihuan Jing \\ Department of Mathematics \\ North Carolina State University, North Carolina, USA \\ jing@math.ncsu.edu \\ Ernest Stitzinger \\ Department of Mathematics \\ North Carolina State University, North Carolina, USA \\ stitz@math.ncsu.edu
}

October 19, 2010

\begin{abstract}
In a recent paper, Carrell and Goulden found a combinatorial identity of the Bernstein operators that they then used to prove Bernstein's Theorem. We show that this identity is a straightforward consequence of the classical result. We also show how a similar approach using the codes of partitions can be generalized from Schur functions to also include Schur $Q$-functions and derive the combinatorial formulation for both cases. We then apply them by examining the LittlewoodRichardson and Pieri Rules.
\end{abstract}

\section{Introduction}

Let $\Lambda=\mathbb{C}\left[p_{1}, p_{2}, p_{3} \ldots\right]=\oplus_{n=0}^{\infty} \Lambda_{n}$ be the ring of symmetric functions, where $p_{n}$ is the power sum symmetric function of degree $n$. As a graded vector 
space, $\Lambda$ has several linear bases such as the power sum symmetric functions $p_{\lambda}$ and Schur functions $s_{\lambda}[\mathrm{M}, \mathrm{St}]$ indexed by partitions. One way to construct Schur functions is to realize them as images of Bernstein operators $B_{n}$, whose generating function $B(t)$ is a variant of vertex operator [Z]:

$$
B(t)=\sum_{n \in \mathbb{Z}} B_{n} t^{n}=\exp \left(\sum_{k \geq 1} \frac{t^{k}}{k} p_{k}\right) \exp \left(-\sum_{k \geq 1} t^{-k} \frac{\partial}{\partial p_{k}}\right)
$$

which acts on the space $\Lambda$. In this construction the Schur function $s_{\lambda}$ is easily given by $s_{\left(\lambda_{1}, \ldots, \lambda_{l}\right)}=B_{\lambda_{1}} \cdots B_{\lambda_{l}} \cdot 1$. The operator $B_{n}$ is a graded linear transformation of degree $n$ defined via its action on the power sum function $p_{\mu}$. We can also define the operator $B_{n}$ on the basis of Schur functions. It turns out the action of $B_{n}$ on Schur functions has a close relationship with Maya diagrams [DJKMO], one of the oldest configurations of partitions. Recently Carrell and Goulden [CG] have formulated the action of $B(t)$ in terms of codes of partitions, which are certain combinatorial description of Maya diagrams. Similar combinatorial structures have also been used in Okounkov's work on random matrices [O].

Carrell and Goulden use codes of partitions to compute the action of Bernstein operators on the Schur function $s_{\lambda}$. They then use this formula to prove Bernstein's Theorem, that $s_{\left(\lambda_{1}, \ldots, \lambda_{l}\right)}=B_{\lambda_{1}} \cdots B_{\lambda_{l}} \cdot 1$ and $B_{m} B_{n}=$ $-B_{n-1} B_{m+1}$. Their proof is combinatorial and they also show that their identity can be used in Plücker relations and KP hierarchies.

In this paper we will show that Carrel and Goulden's formula can be easily obtained from the classical results using algebraic properties satisfied by vertex operators. We will also generalize the combinatorial structures to the case of Schur $Q$-functions and derive a similar but simpler combinatorial formulation for the associated vertex operator. The Schur $Q$-functions are certain distinguished linear bases in the subring of symmetric functions:

$$
\Lambda^{-}=\mathbb{C}\left[p_{1}, p_{3}, p_{5} \ldots\right]=\oplus_{n=0}^{\infty} \Lambda_{n}^{-} .
$$

These symmetric functions were defined by I. Schur in his seminal work [Sch] on projective representations of the symmetric group $S_{n}$ (see also [M]). As pointed out in [J1] Schur functions and Schur $Q$-functions are two examples of the celebrated Boson-Fermion correspondence, in which they can be roughly viewed as untwisted and twisted pictures of the Fock space representations respectively, and the vertex operators for Schur and Schur $Q$-functions 
come from two different realizations of affine Lie algebras. Taking the advantage of this grand picture we can give a unified approach to derive the action of vertex operators on Schur and Schur $Q$-functions.

First we can compute the action of Bernstein operator by using the commutation relations:

$$
B_{m} B_{n}=-B_{n-1} B_{m+1} .
$$

The combinatorial structure of codes then follows easily from the algebraic structure.

When we tensor the ring $\Lambda$ by the group algebra of one-dimensional lattice $\mathbb{Z}$, the commutation relations (11) can be improved into the exact anticommutation relations of the vertex operators $X(t)$ :

$$
X_{m} X_{n}=-X_{n} X_{m},
$$

thus we obtain our second and even simpler proof of Carrell-Goulden's formula. Using the same idea we can generalize this to the twisted Fock space $\Lambda^{-}=\mathbb{C}\left[p_{1}, p_{3}, \ldots\right]$ and again we use the similar antisymmetry of the components of the vertex operator $Y(z)$ (see [J1]) to study the action of the Schur $Q$-functions.

We also formulate the action of the twisted vertex operators in terms of shifted codes. In this way we have unified codes and shifted codes in the context of vertex operators and Boson-Fermion correspondence. We also show how these combinatorial objects can help us derive the LittlewoodRichardson Rule and the Pieri Rules.

\section{Codes of Partitions}

Let $\lambda=\left(\lambda_{1}, \lambda_{2}, \ldots, \lambda_{l}\right)$ be a decreasing sequence of positive integers, $\lambda_{1} \geq$ $\lambda_{2} \geq \cdots \geq \lambda_{l}>0$. We say that $\lambda$ is a partition of $n$, denoted $\lambda \vdash n$, if $\lambda_{1}+\lambda_{2}+\cdots+\lambda_{l}=n$. We also say that the weight of the partition $\lambda$ is $|\lambda|=n$, and the length of the partition is $l(\lambda)=l$.

The Young diagram of a partition $\lambda$ is the left-justified arrangement of boxes with $\lambda_{i}$ boxes in the $i^{\text {th }}$ row from the top. Since the parts of $\lambda$ are weakly decreasing, the number of boxes in each row will be less than or equal to the number of boxes in each row above it.

Define the code of a partition $\lambda$ to be the doubly infinite sequence of letters $\mathrm{R}$ and $\mathrm{U}$ obtained from the Young diagram of shape $\lambda$ as follows. 
Consider the Young diagram top and left aligned in the $4^{\text {th }}$ quadrant of the $x y$-plane together with the negative $y$-axis and the positive $x$-axis. Trace up the negative $y$-axis to the bottom of the Young diagram, then along the bottommost edge of the Young diagram, then right along the positive $x$-axis. The code of the partition is the sequence of R's and U's obtained from this path, where $\mathrm{R}$ corresponds to a unit right step and $\mathrm{U}$ corresponds to a unit up step.

Example 2.1. Let $\lambda=(4,2,1)$. Then the Young diagram of shape $\lambda$ in the $4^{\text {th }}$ quadrant of the $x y$-plane is shown below, with the path described above in bold.

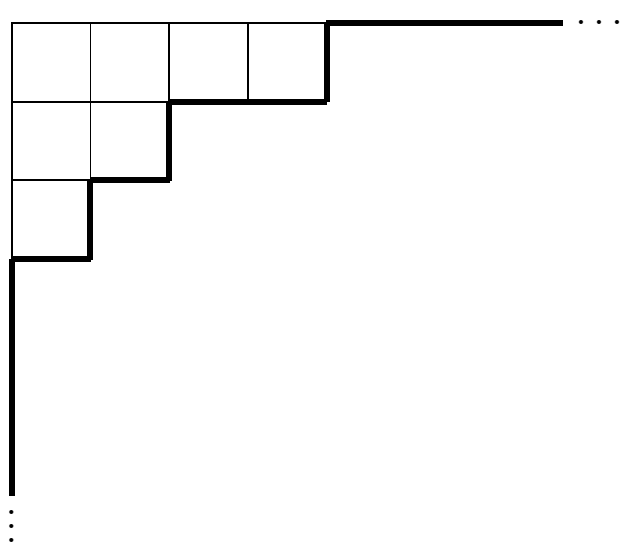

The path consists of infinitely many U's at the beginning - corresponding to tracing up the negative $y$-axis, then RURURRU - corresponding to tracing the bottommost edge of the Young diagram, then infinitely many R's at the end - corresponding to tracing right along the positive $x$-axis. Thus the sequence ... UUURURURRURRR ... is the code of the partition $\lambda=$ $(4,2,1)$.

Note that the code of any partition will always have infinitely many U's at the beginning of the code, and infinitely many R's at the end of the code (corresponding respectively to the negative $y$-axis and the positive $x$-axis).

Define the partition $\lambda^{(i)}$ to be the partition obtained by turning the $i^{\text {th }} \mathrm{R}$ from the left in the code of $\lambda$ to a U. Equivalently, $\lambda^{(i)}$ is the partition obtained by looking at the lower-right edge of the associated Young diagram (together with the positive $x$-axis and negative $y$-axis, where the Young diagram is considered to be in the $4^{\text {th }}$ quadrant) and turning the $i^{\text {th }}$ horizontal edge 
from the left into a vertical edge (and shifting the resulting path into the $4^{\text {th }}$ quadrant).

Example 2.2. Let $\lambda=(4,2,1)$. Then to find $\lambda^{(3)}$, the third right step from the left becomes an up step. The changed edge is shown in bold below.
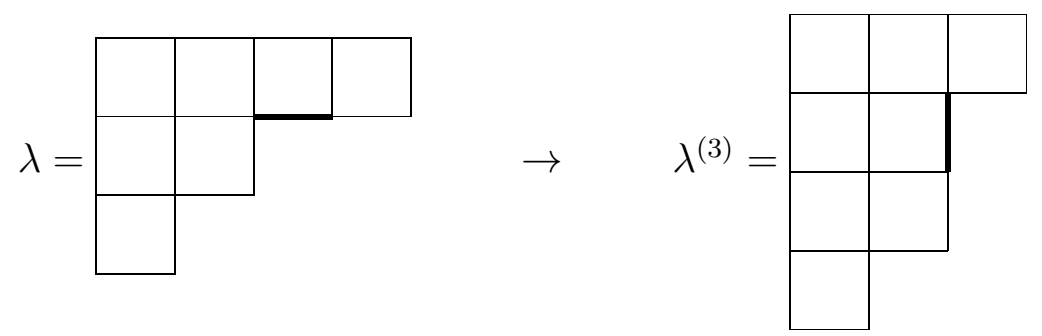

Thus $\lambda^{(3)}=(3,2,2,1)$.

A simple formula for the partition $\lambda^{(i)}$ is

$$
\lambda^{(i)}=\left(\lambda_{1}-1, \lambda_{2}-1, \ldots, \lambda_{j}-1, i-1, \lambda_{j+1}, \ldots, \lambda_{l}\right),
$$

where $\lambda_{j} \geq i>\lambda_{j+1}$ (with the convention that $\lambda_{l+1}=0$ and $\lambda_{0}=\infty$ ).

Given the code of a partition $\lambda$, let $u_{i}(\lambda)$ be the number of U's in the code of $\lambda$ to the right of the $i^{\text {th }} \mathrm{R}$ from the left, and let $r_{i}(\lambda)$ be the number of R's in the code of $\lambda$ to the left of the $i^{\text {th }} \mathrm{U}$ from the right. This means that $u_{i}(\lambda)$ is equal to the number of parts of $\lambda$ of size at least $i$, and $r_{i}(\lambda)=\lambda_{i}$.

\section{Bernstein Operators}

Recall that the Bernstein operators $B(t)$, and $B_{n}$ are given by

$$
B(t)=\sum_{n \in \mathbb{Z}} B_{n} t^{n}=\exp \left(\sum_{k \geq 1} \frac{t^{k}}{k} p_{k}\right) \exp \left(-\sum_{k \geq 1} t^{-k} \frac{\partial}{\partial p_{k}}\right),
$$

which acts on the ring of polynomials $\Lambda=\mathbb{C}\left[p_{1}, p_{2}, p_{3}, \ldots\right]$. Here the power sum $p_{n}$ acts as a multiplication on $\Lambda$. Bernstein's primary result with these operators was Bernstein's formula, which states:

$$
s_{\lambda}=B_{\lambda_{1}} B_{\lambda_{2}} \cdots B_{\lambda_{l}} \cdot 1,
$$


where $\lambda=\left(\lambda_{1}, \lambda_{2}, \ldots, \lambda_{l}\right)$, and $s_{\lambda}$ is the Schur polynomial indexed by $\lambda$. For convenience, we will often denote this composition as $B_{\lambda_{1}} B_{\lambda_{2}} \cdots B_{\lambda_{l}} \cdot 1=$ $B_{\lambda_{1}, \lambda_{2}, \ldots, \lambda_{l}} \cdot 1$. Another key relation satisfied by Bernstein operators is the following:

$$
B_{n} B_{m}=-B_{m-1} B_{n+1} .
$$

We will now use these two results, Eqs.(4) and (5), to prove a formula given in [CG which gives the action of Bernstein's operators on the Schur polynomials.

Theorem 3.1. For any partition $\lambda$,

$$
B(t) s_{\lambda}=\sum_{i \geq 1}(-1)^{|\lambda|-\left|\lambda^{(i)}\right|+i-1} t^{\left|\lambda^{(i)}\right|-|\lambda|} s_{\lambda^{(i)}} .
$$

This result was originally proved in [CG using some combinatorial considerations and the dual action of the Schur functions. We would like to give two simpler proofs to this result, which will motivate our later generalization to the case of the Schur $Q$-functions.

Proof: Since $B(t)=\sum_{n \in \mathbb{Z}} B_{n} t^{n}$, we only need to determine the action of $B_{n}$ on $s_{\lambda}$. By equation (4),

$$
B_{n} s_{\lambda}=B_{n} B_{\lambda_{1}} B_{\lambda_{2}} \cdots B_{\lambda_{l}} \cdot 1
$$

Case 1: If $n \geq \lambda_{1}$, then by equation (41),

$$
B_{n, \lambda_{1}, \lambda_{2}, \ldots, \lambda_{l}} \cdot 1=s_{\left(n, \lambda_{1}, \lambda_{2}, \ldots, \lambda_{l}\right)}=s_{\lambda^{(n+1)}},
$$

where this term in the summation on the right has a $t$ term of $\left|\lambda^{(n+1)}\right|-|\lambda|$. Since $n \geq \lambda_{1}$, turning the $(n+1)^{\text {th }}$ horizontal edge to a vertical edge creates a new first row of size $n$. So $\left|\lambda^{(n+1)}\right|-|\lambda|=n$ which is the exponent of $t$ associated with $B_{n}$.

Case 2: If $n=\lambda_{j}-j$ for some $j, 1 \leq j \leq l$, then by equation (5),

$$
\begin{aligned}
B_{n, \lambda_{1}, \lambda_{2}, \ldots, \lambda_{l}} \cdot 1 & =(-1) B_{\lambda_{1}-1, n+1, \lambda_{2}, \ldots, \lambda_{l}} \cdot 1 \\
& =(-1)^{2} B_{\lambda_{1}-1, \lambda_{2}-1, n+2, \lambda_{3}, \ldots, \lambda_{l}} \cdot 1 \\
& \vdots \\
& =(-1)^{j-1} B_{\lambda_{1}-1, \lambda_{2}-1, \ldots, \lambda_{j-1}-1, n+j-1, \lambda_{j}, \lambda_{j+1}, \ldots, \lambda_{l}} \cdot 1,
\end{aligned}
$$


but $n=\lambda_{j}-j$, so $n+j-1=\lambda_{j}-1$. From equation (5), $B_{i, i+1}=-B_{i, i+1}$ for all $i$, which implies $B_{i, i+1}=0$ for all $i$. Since $B_{n+j-1, \lambda_{j}}=B_{\lambda_{j}-1, \lambda_{j}}$ is such a term, this product is zero.

Case 3: If $\lambda_{j+1}-(j+1)<n<\lambda_{j}-j$ for some $j, 1 \leq j<l$, then similarly,

$$
\begin{aligned}
B_{n, \lambda_{1}, \lambda_{2}, \ldots, \lambda_{l}} \cdot 1 & =(-1)^{j} B_{\lambda_{1}-1, \lambda_{2}-1, \ldots, \lambda_{j}-1, n+j, \lambda_{j+1}, \ldots, \lambda_{l}} \cdot 1 \\
& =(-1)^{j} s_{\left(\lambda_{1}-1, \lambda_{2}-1, \ldots, \lambda_{j}-1, n+j, \lambda_{j+1}, \ldots, \lambda_{l}\right)} \\
& =(-1)^{(-n)+(n+j+1)-1} s_{\lambda^{(n+j+1)}} \\
& =(-1)^{\left(|\lambda|-\left|\lambda^{(n+j+1)}\right|\right)+(n+j+1)-1} s_{\lambda^{(n+j+1)}},
\end{aligned}
$$

by equation (41), since $\lambda_{1}-1 \geq \lambda_{2}-1 \geq \cdots \geq \lambda_{j}-1 \geq n+j \geq \lambda_{j+1} \geq \cdots \geq \lambda_{l}$. Note that $|\lambda|-\left|\lambda^{(n+j+1)}\right|=-n$ since $\lambda^{(n+j+1)}$ removes the last box from each of the first $j$ rows of $\lambda$ 's Young diagram and then adds a row of size $n+j$. Also note that the exponent of $t$ associated with $s_{\lambda^{(n+j+1)}}$ is $\left|\lambda^{(n+j+1)}\right|-|\lambda|=n$, the same exponent associated with $B_{n}$.

Case 4: If $n<\lambda_{l}-l$, then similarly,

$$
B_{n, \lambda_{1}, \lambda_{2}, \ldots, \lambda_{l}} \cdot 1=(-1)^{l} B_{\lambda_{1}-1, \lambda_{2}-1, \ldots, \lambda_{l}-1, n+l} \cdot 1 .
$$

- If $n+l \geq 0$, then by equation (41),

$$
\begin{aligned}
(-1)^{l} B_{\lambda_{1}-1, \lambda_{2}-1, \ldots, \lambda_{l}-1, n+l} \cdot 1 & =(-1)^{(-n)+(n+l+1)-1} s_{\lambda^{(n+l+1)}} \\
& =(-1)^{|\lambda|-\left|\lambda^{(n+l+1)}\right|+(n+l+1)-1} s_{\lambda^{(n+l+1)}},
\end{aligned}
$$

because $|\lambda|-\left|\lambda^{(n+l+1)}\right|=-n$ since $\lambda^{(n+l+1)}$ removes the last box from each of the $l$ rows of $\lambda$ 's Young diagram and then adds a row of size $n+l$. Again note that the exponent of $t$ associated with $s_{\lambda^{(n+l+1)}}$ is $\left|\lambda^{(n+l+1)}\right|-|\lambda|=n$, the same exponent associated with $B_{n}$.

- If $n+l<0$, then by equation (5):

$$
\begin{gathered}
B_{-1,0}=-B_{-1,0}=0 \\
B_{-a, 0}=-B_{-1,-a+1}=B_{-1,-1,-a+2}=\cdots=(-1)^{a} B_{-1,-1, \ldots,-1,0}=0,
\end{gathered}
$$

for all $a \in \mathbb{Z}^{+}$, since $B_{0} \cdot 1=1$. This implies that

$$
(-1)^{l} B_{\lambda_{1}-1, \lambda_{2}-1, \ldots, \lambda_{l}-1, n+l} \cdot 1=(-1)^{l} B_{\lambda_{1}-1, \lambda_{2}-1, \ldots, \lambda_{l}-1,-a} \cdot 1=0 .
$$


This proves the theorem.

We can also prove this theorem using vertex operators. This method will be particularly interesting to us because the same approach can be used to analyze the Schur $Q$-functions.

To see the symmetry of the indices of the Schur functions, we use a modified version of Bernstein's operator from $[\mathrm{J} 1$. Let $\mathbb{C}[\mathbb{Z}]$ be the group algebra of $\mathbb{Z}$ generated by $e^{p}$, meaning $\mathbb{C}[\mathbb{Z}]=\oplus_{n \in \mathbb{Z}} \mathbb{C} e^{n p}$. Consider the two operators $e^{p}$ and $t^{\partial_{p}}$ on $\mathbb{C}[\mathbb{Z}]$ defined by

$$
\begin{aligned}
e^{p} \cdot e^{n p} & =e^{(n+1) p} \\
t^{\partial_{p}} \cdot e^{n p} & =t^{n} e^{n p} .
\end{aligned}
$$

Following [J1], the vertex operator $X(t)$ is defined on $\Lambda \otimes \mathbb{C}[\mathbb{Z}]$ by

$$
X(t)=B\left(t^{-1}\right) e^{p} t^{\partial_{p}}=\sum_{n \in \mathbb{Z}} X_{n} t^{-n} .
$$

The following result was proved in [J2]: the product of the vertex operator $X(t)$ is antisymmetric, so $X_{n} X_{m}=-X_{m} X_{n}$, and we have the following theorem, which is a modified version from [J2].

Theorem 3.2. 1. For any $l \in \mathbb{N}$, one has

$$
X_{t_{1}} \cdots X_{t_{l}}=(-1)^{l(\sigma)} X_{t_{\sigma(1)}} \cdots X_{t_{\sigma(l)}},
$$

for all $\sigma$ in $S_{l}$, where $l(\sigma)$ is the number of inversions in the permutation $\sigma$.

2. For any partition $\mu=\left(\mu_{1}, \ldots, \mu_{l}\right)$, we have

$$
X_{-\mu_{1}} \cdots X_{-\mu_{l}} \cdot e^{m p}=s_{\mu-\delta+l \mathbb{\mathbb { N }}} e^{(m+l) p},
$$

where $\delta=(l-1, \ldots, 2,1,0)$ and $\mathbb{1}=(1, \ldots, 1) \in \mathbb{N}^{l}$.

In particular, this means that

$$
X_{-\mu_{1}} \cdots X_{-\mu_{l}} \cdot e^{-l p}=s_{\mu-\delta+l \mathbb{1}} .
$$

For simplicity, we removed the index shift of $\frac{1}{2}$ in the definition of $X(t)$ (see [J1, J2]). 
Now we can give a simpler proof of Theorem 3.1. For simplicity, we will denote the composition as $X_{-\mu_{1}} X_{-\mu_{2}} \cdots X_{-\mu_{l}}=X_{-\mu_{1},-\mu_{2}, \ldots,-\mu_{l}}$.

$$
\begin{aligned}
B(t) s_{\lambda} & =B(t) X_{-\left(\lambda_{1}-1\right),-\left(\lambda_{2}-2\right), \ldots,-\left(\lambda_{l}-l\right)} \cdot e^{-l p} \\
& =X(t)\left(e^{p} t^{\partial_{p}}\right)^{-1} X_{-\left(\lambda_{1}-1\right),-\left(\lambda_{2}-2\right), \ldots,-\left(\lambda_{l}-l\right)} \cdot e^{-l p} \\
& =X(t) X_{-\left(\lambda_{1}-2\right),-\left(\lambda_{2}-3\right), \ldots,-\left(\lambda_{l}-l-1\right)} \cdot\left(e^{p} t^{\partial_{p}}\right)^{-1} t^{-l} e^{-l p} \\
& =X(t) X_{-\left(\lambda_{1}-2\right),-\left(\lambda_{2}-3\right), \ldots,-\left(\lambda_{l}-l-1\right)} \cdot e^{-(l+1) p} \\
& =\sum_{n \in \mathbb{Z}} X_{-n} X_{-\left(\lambda_{1}-2\right),-\left(\lambda_{2}-3\right), \ldots,-\left(\lambda_{l}-l-1\right)} \cdot e^{-(l+1) p} t^{n} \\
& =\sum_{n \neq \lambda_{k}-k-1}(-1)^{j} X_{-\left(\lambda_{1}-2\right), \ldots,-\left(\lambda_{j}-j-1\right),-n,-\left(\lambda_{j+1}-j-2\right), \ldots,-\left(\lambda_{l}-l-1\right)} \cdot e^{-(l+1) p} t^{n} \\
& =\sum_{n \neq \lambda_{k}-k-1}(-1)^{j} X_{-\left(\lambda_{1}^{(i)}-1\right), \ldots,-\left(\lambda_{l+1}^{(i)}-l-1\right)} \cdot e^{-(l+1) p} t^{n} s_{\lambda^{(i)},},
\end{aligned}
$$

where $\lambda_{j}-j-1>n>\lambda_{j+1}-j-2$ and $i=n+j+1$, so $\lambda_{j}>i \geq \lambda_{i+1}-1$. This definition of $i$ also implies that $n=\left|\lambda^{(i)}\right|-|\lambda|, j=|\lambda|-\left|\lambda^{(i)}\right|+i-1$, and $\lambda^{(i)}=$ $\left(\lambda_{1}-1, \ldots, \lambda_{j}-1, i-1, \lambda_{j+1}, \ldots, \lambda_{l}\right)=\left(\lambda_{1}-1, \ldots, \lambda_{j}-1, n+j, \lambda_{j+1}, \ldots, \lambda_{l}\right)$. With this identification this last line becomes the following:

$$
B(t) s_{\lambda}=\sum_{i \geq 1}(-1)^{|\lambda|-\left|\lambda^{(i)}\right|+i-1} t^{\left|\lambda^{(i)}\right|-|\lambda|} s_{\lambda^{(i)}}
$$

and Theorem 3.1 is proved.

\section{Schur Q-function}

We will next state and prove a similar result for the Schur $Q$-functions, $Q_{\lambda}$, where $\lambda$ is a strict partition, i.e. $\lambda_{1}>\lambda_{2}>\cdots>\lambda_{l}$ and $\lambda_{i} \in \mathbb{N}$.

For any partition $\mu=\left(1^{m_{1}(\mu)} 2^{m_{1}(\mu)} \ldots\right)$, we define $z_{\mu}=\prod_{i \geq 1} i^{m_{i}(\mu)} m_{i}(\mu)$ !. We consider the ring of symmetric functions in $x_{1}, x_{2}, \ldots$, but restrict ourselves to polynomials in odd degree power sums

$$
p_{2 k+1}=\sum_{i \geq 1} x_{i}^{2 k+1}, \quad k \in \mathbb{Z}_{+} .
$$

Let $\mathcal{O} P$ denote the set of partitions with odd parts, and let $\Lambda^{-}$be the ring of symmetric functions generated by $p_{2 k+1}, k \in \mathbb{Z}_{+}$. Under the inner product

$$
<p_{\lambda}, p_{\mu}>=2^{-l(\lambda)} \delta_{\lambda, \mu} z_{\lambda}, \quad \lambda, \mu \in \mathcal{O P},
$$


the space $\Lambda^{-}$has $Q_{\lambda}$ ( $\lambda$ strict) as a distinguished orthogonal basis of symmetric polynomials [Sch, $\mathrm{M}]$. They play a fundamental role in the construction of projective representations of the symmetric group $S_{n}$.

On the space $\Lambda^{-}$we recall the definition of the twisted vertex operator JI1:

$$
Y(t)=\sum_{n \in \mathbb{Z}} Y_{n} t^{-n}=\exp \left(\sum_{k \geq 1} \frac{2 t^{-2 k+1}}{k} p_{2 k-1}\right) \exp \left(-\sum_{k \geq 1} t^{2 k-1} \frac{\partial}{\partial p_{2 k-1}}\right),
$$

which acts on the ring of polynomials $\Lambda^{-}=\mathbb{C}\left[p_{1}, p_{3}, p_{5}, \ldots\right]$, and the power sum $p_{2 k-1}$ acts as a multiplication on $\Lambda^{-}$.

From [J1, we have that the following two results hold:

$$
Q_{\lambda}=Y_{-\lambda_{1}} Y_{-\lambda_{2}} \cdots Y_{-\lambda_{l}} \cdot 1
$$

where $\lambda=\left(\lambda_{1}, \lambda_{2}, \ldots, \lambda_{l}\right)$, and $Q_{\lambda}$ is the Schur $Q$-function indexed by $\lambda$. Again, we will often denote this composition as $Y_{\lambda_{1}} Y_{\lambda_{2}} \cdots Y_{\lambda_{l}} \cdot 1=Y_{\lambda_{1}, \lambda_{2}, \ldots, \lambda_{l}} \cdot 1$. The second result is:

$$
Y_{n} Y_{m}=-Y_{m} Y_{n}
$$

Theorem 4.1. For any strict partition $\lambda$,

$$
Y(t) Q_{\lambda}=\sum_{n \neq \lambda_{j}}(-1)^{i} t^{n} Q_{\left(\lambda_{1}, \lambda_{2}, \ldots, \lambda_{i}, n, \lambda_{i+1}, \ldots, \lambda_{l}\right)} .
$$

Proof: Recall that $Y(t)=\sum_{n \in \mathbb{Z}} Y_{n} t^{-n}$, then use equations (66) and (77):

$$
\begin{aligned}
Y(t) Q_{\lambda}=\sum_{n \in \mathbb{Z}} Y_{n} t^{-n} Q_{\lambda} & =\sum_{n \in \mathbb{Z}} t^{n} Y_{-n} Y_{-\lambda_{1}} Y_{-\lambda_{2}} \cdots Y_{-\lambda_{l}} \cdot 1 \\
& =\sum_{n \neq \lambda_{j}}(-1)^{i} t^{n} Y_{-\lambda_{1},-\lambda_{2},-\ldots,-\lambda_{i},-n,-\lambda_{i+1}, \ldots,-\lambda_{l}} \cdot 1 \\
& =\sum_{n \neq \lambda_{j}}(-1)^{i} t^{n} Q_{\left(\lambda_{1}, \lambda_{2}, \ldots, \lambda_{i}, n, \lambda_{i+1}, \ldots, \lambda_{l}\right)},
\end{aligned}
$$

where $\lambda_{i}>n>\lambda_{i+1}$, because by equation (77), $Y_{-n} Y_{-\lambda_{j}}=Y_{-n} Y_{-n}=0$ if $n=\lambda_{j}$.

We can also interpret the result in terms of codes of strict partitions, but we first need to reinterpret how codes behave for strict partitions. 
Definition 4.2. Define the partition $\lambda^{[i]}$ to be the partition obtained from the code of a strict partition $\lambda$ by inserting a $\mathrm{U}$ between the $i^{\text {th }}$ pair of consecutive R's (with the convention that three consecutive R's counts as two pairs, four consecutive R's counts as three pairs, and so on). Equivalently, $\lambda^{[i]}$ is the partition obtained from the code of $\lambda$ by inserting a $\mathrm{U}$ after the $i^{\text {th }} \mathrm{R}$ which is immediately followed by an R.

Example 4.3. For example, if $\lambda=(6,4,3,1)$, the first pair of consecutive R's in the code of $\lambda$ is shown below in bold, with the new edge inserted between them to get $\lambda^{[1]}$ also shown in bold.

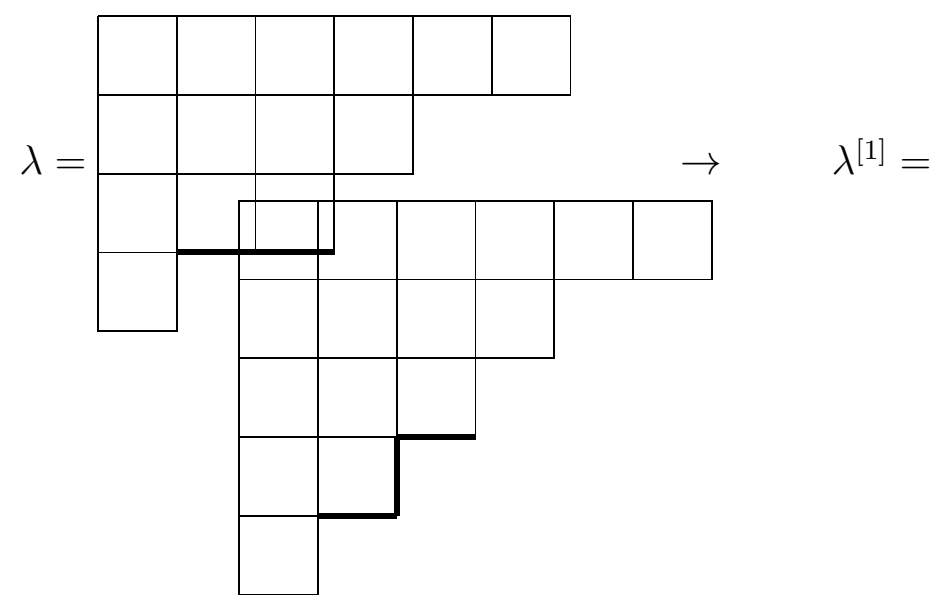

To get $\lambda^{[2]}$, we insert a $\mathrm{U}$ between the second pair of consecutive R's in the code of $\lambda$. Again the pair of right steps corresponding to those R's are shown below in bold, along with the up step inserted between them.

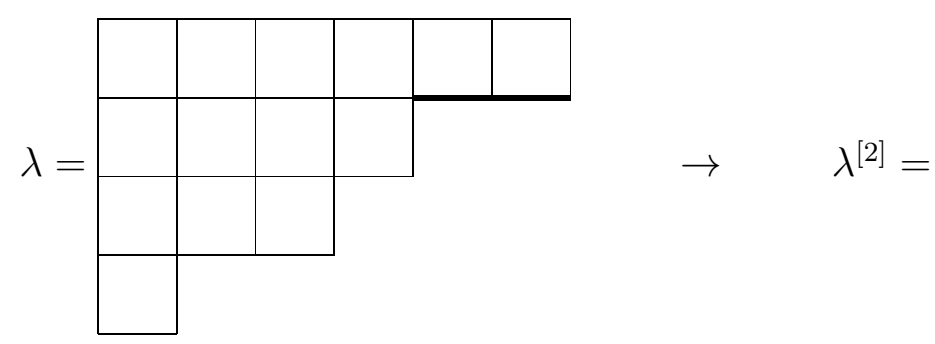




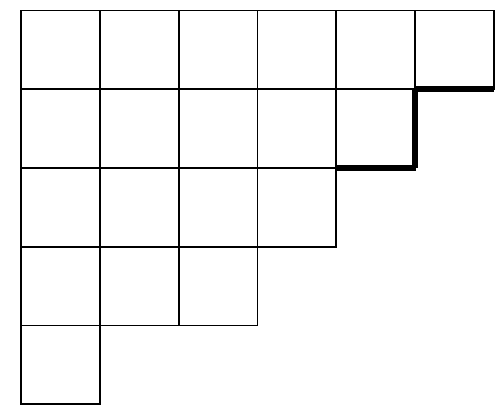

To get $\lambda^{[3]}$, we insert a $\mathrm{U}$ between the third pair of consecutive R's in the code of $\lambda$. This works the same way as the previous examples, except that the third pair of R's are in the part of the code corresponding to the positive $x$-axis. Again the pair of right steps corresponding to those R's are shown below in bold, along with the up step inserted between them.

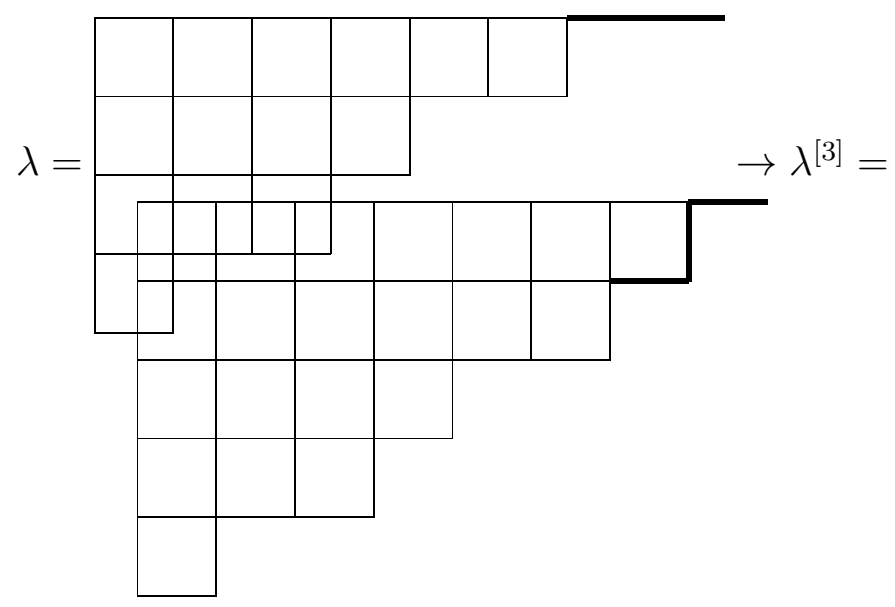

Another way to think about $\lambda^{[i]}$ is the following. With this definition $\lambda^{[i]}$ is the strict partition with the $i^{\text {th }}$ smallest possible integer inserted into the partition $\lambda$. This means that $\lambda^{[1]}$ is the strict partition with the smallest possible integer inserted into $\lambda$. For $\lambda=(6,4,3,1)$, the smallest integer that can be inserted to still have a strict partition is 2 , so $\lambda^{[1]}=(6,4,3,2,1)$. The second smallest integer that can be inserted into $\lambda=(6,4,3,1)$ is 5 , so 
$\lambda^{[1]}=(6,5,4,3,1)$. Similarly, $\lambda^{[3]}=(7,6,4,3,1), \lambda^{[4]}=(8,6,4,3,1)$, and so on.

Often strict partitions are associated with shifted Young diagram [Ste] rather than Young diagram. A shifted Young diagram of shape $\lambda$, where $\lambda$ is a strict partition, is an arrangement of boxes with $\lambda_{i}$ boxes in the $i^{\text {th }}$ row, with the leftmost box in each row one unit to the right of the leftmost box of the row above it. This is sometimes more intuitive since the rightmost edge of a shifted Young diagram of shape $\lambda$, where $\lambda$ is a strict partition, follows the same rules of a Young diagram of shape $\mu$, where $\mu$ is any partition, namely that the rightmost edge moves weakly left as you go from top to bottom. We can use this correlation to reinterpret $\lambda^{[i]}$ using the analogue of our existing machinery for codes on a shifted Young diagram of shape $\lambda$.

Definition 4.4. Define the shifted code of a strict partition $\lambda$ to be the infinite sequence of letters $\mathrm{R}$ and $\mathrm{U}$ obtained from the shifted Young diagram of shape $\lambda$ as follows. Consider the shifted Young diagram top and left aligned in the $4^{\text {th }}$ quadrant of the $x y$-plane together with the positive $x$-axis. Starting at the bottom right corner of the leftmost box in the last row, trace along the rightmost edge of the shifted Young diagram, then right along the positive $x$-axis. Equivalently, start the code at the lowest place where the line $y=-x$ intersects the shifted Young diagram. The shifted code of the strict partition is the sequence of R's and U's obtained from this path, where R corresponds to a unit right step and $\mathrm{U}$ corresponds to a unit up step.

Example 4.5. Let $\lambda=(5,4,2)$. Then the shifted Young diagram of shape $\lambda$ in the $4^{\text {th }}$ quadrant of the $x y$-plane is shown below, with the path described above in bold.

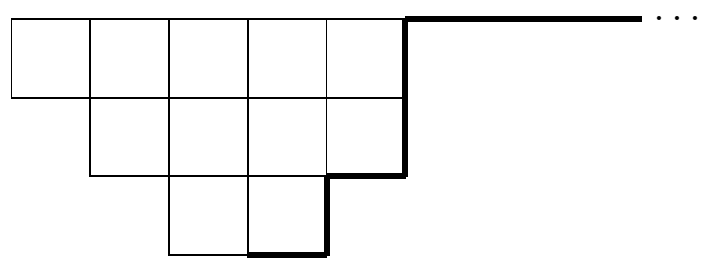

Example 4.6. Let $\lambda=(6,4,3,1)$. Then the shifted Young diagram of shape $\lambda$ in the $4^{\text {th }}$ quadrant of the $x y$-plane is shown below, with the path described above in bold. 


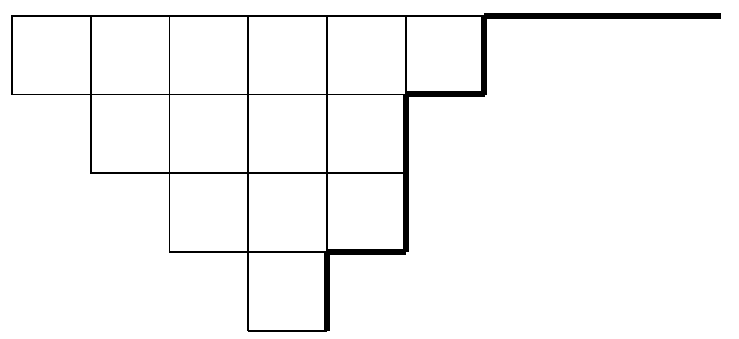

Note that the shifted code is not doubly infinite like the code of an arbitrary partition, since it has a fixed starting point. It does however retain the property that there are infinitely many R's at the end of the code.

Using shifted codes we can reinterpret our definition of $\lambda^{[i]}$. For a strict partition $\lambda, \lambda^{[i]}$ is obtained from the shifted code of $\lambda$ by turning the $i^{\text {th }} \mathrm{R}$ in the shifted code to a $\mathrm{U}$. This is since either method inserts the $i^{\text {th }}$ smallest possible integer into the partition $\lambda$ to still have a strict partition, or since the number of pairs of consecutive R's between two U's is the number of consecutive R's minus one, which is the number of R's in the shifted code corresponding to the same row.

Example 4.7. We return to our example $\lambda=(6,4,3,1)$. Then we can find $\lambda^{[2]}$ by turning the second right step from the left in the shifted code of $\lambda$ into an up step. The changed edge is shown in bold below.

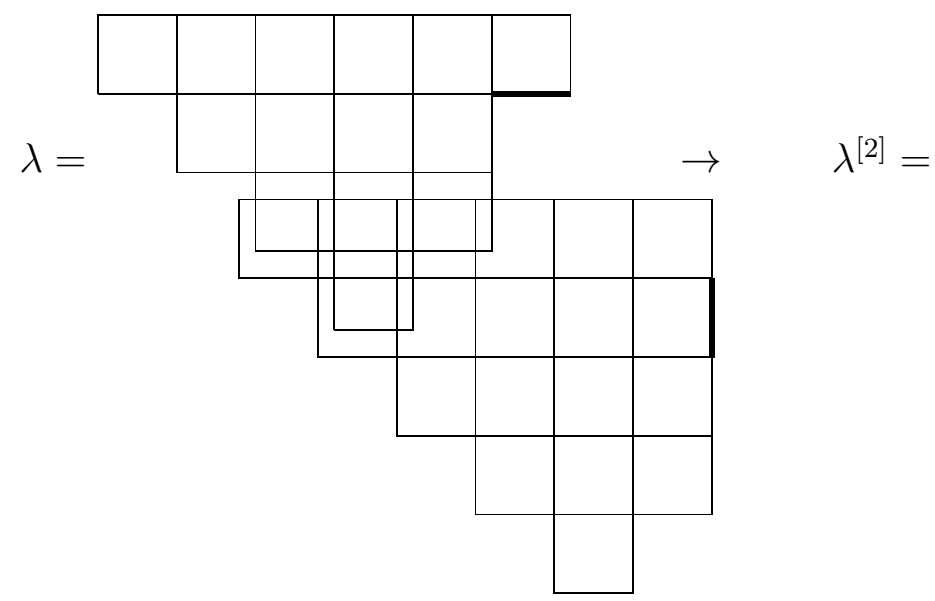


Given the code of a strict partition $\lambda$, let $\tilde{u}_{i}(\lambda)$ be the number of U's in the code of $\lambda$ to the right of the $i^{\text {th }}$ pair of consecutive R's from the left, which is the number of U's in the shifted code of $\lambda$ to the right of the $i^{\text {th }} \mathrm{R}$ from the left.

This means that $\tilde{u}_{i}(\lambda)$ is equal to the number of parts of $\lambda$ greater than the $i^{\text {th }}$ smallest possible integer that can be inserted into $\lambda$, which is equal to the number of parts of $\lambda$ of size at least $\left|\lambda^{[i]}\right|-|\lambda|$. Then the number of parts of $\lambda$ of size at least $\left|\lambda^{[i]}\right|-|\lambda|$ is the length of $\lambda$ minus the number of parts of size less than $\left|\lambda^{[i]}\right|-|\lambda|$. But the number of parts less than $\left|\lambda^{[i]}\right|-|\lambda|$ is the number of integers less than $\left|\lambda^{[i]}\right|-|\lambda|$ minus the number of integers less than $\left|\lambda^{[i]}\right|-|\lambda|$ that are not in $\lambda$, which is $\left(\left|\lambda^{[i]}\right|-|\lambda|-1\right)-(i-1)=$ $\left|\lambda^{[i]}\right|-|\lambda|-i$. So $\tilde{u}_{i}(\lambda)=l(\lambda)-\left(\left|\lambda^{[i]}\right|-|\lambda|-i\right)=l(\lambda)+|\lambda|-\left|\lambda^{[i]}\right|+i$.

We can now use $\lambda^{[i]}$ to reinterpret Theorem 4.1.

Theorem 4.8. For any strict partition $\lambda$,

$$
Y(t) Q_{\lambda}=\sum_{i \geq 1}(-1)^{l(\lambda)+|\lambda|-\left|\lambda^{[i]}\right|+i} t^{\left|\lambda^{[i]}\right|-|\lambda|} Q_{\lambda^{[i]}}
$$

Proof: By Theorem 4.1, we know that

$$
Y(t) Q_{\lambda}=\sum_{n \neq \lambda_{j}}(-1)^{k} t^{n} Q_{\left(\lambda_{1}, \lambda_{2}, \ldots, \lambda_{k}, n, \lambda_{k+1}, \ldots, \lambda_{l}\right)} .
$$

But $\lambda^{[i]}$ is the partition with the $i^{\text {th }}$ smallest possible integer that can be inserted into the partition $\lambda$. Thus $\left(\lambda_{1}, \lambda_{2}, \ldots, \lambda_{k}, n, \lambda_{k+1}, \ldots, \lambda_{l}\right)=\lambda^{[i]}$ for some $i$, where $n$ is the $i^{\text {th }}$ smallest possible integer that can be inserted into $\lambda$, so $n=\left|\lambda^{[i]}\right|-|\lambda|$. Then $k$ is the number of parts of $\lambda$ greater than the $i^{\text {th }}$ smallest possible integer that can be inserted into $\lambda$, so by definition $k=\tilde{u}_{i}(\lambda)$. Thus

$$
\begin{aligned}
Y(t) Q_{\lambda} & =\sum_{n \neq \lambda_{j}}(-1)^{k} t^{n} Q_{\left(\lambda_{1}, \lambda_{2}, \ldots, \lambda_{k}, n, \lambda_{k+1}, \ldots, \lambda_{l}\right)} \\
& =\sum_{i \geq 1}(-1)^{\tilde{u}_{i}(\lambda)} t^{\left|\lambda^{[i]}\right|-|\lambda|} Q_{\lambda^{[i]}} \\
& =\sum_{i \geq 1}(-1)^{l(\lambda)+|\lambda|-\left|\lambda^{[i]}\right|+i} t^{\left|\lambda^{[i]}\right|-|\lambda|} Q_{\lambda^{[i]}}
\end{aligned}
$$

since we know $\tilde{u}_{i}(\lambda)=k=l(\lambda)+|\lambda|-\left|\lambda^{[i]}\right|+i,\left|\lambda^{[i]}\right|-|\lambda|=n$, and $\lambda^{[i]}=\left(\lambda_{1}, \lambda_{2}, \ldots, \lambda_{i}, n, \lambda_{i+1}, \ldots, \lambda_{l}\right)$. 


\section{Littlewood-Richardson Rule}

One application for codes of partitions is the following theorem, which gives a new way to compute Littlewood-Richardson coefficients [M, Sa], using only the codes of the partitions involved.

A skew-partition $\lambda / \mu$ is a horizontal $n$-strip if no column in the Young diagram of $\lambda / \mu$ has more than one box. Equivalently, $\lambda / \mu$ is a horizontal $n$-strip if $\lambda_{i+1} \leq \mu_{i} \leq \lambda_{i}$ for all $1 \leq i \leq l(\mu)$, where $l(\mu)$ is the length of $\mu$.

A skew-partition $\lambda / \mu$ is a vertical $n$-strip if no row in the Young diagram of $\lambda / \mu$ has more than one box. Equivalently, $\lambda / \mu$ is a horizontal $n$-strip if $\lambda_{i}-1 \leq \mu_{i} \leq \lambda_{i}$ for all $1 \leq i \leq l(\mu)$, where $l(\mu)$ is the length of $\mu$.

Theorem 5.1. (The Littlewood-Richardson Rule)

$$
s_{\mu} s_{\nu}=\sum_{\lambda} c_{\mu, \nu}^{\lambda} s_{\lambda}=\sum_{\left(\mu=\mu^{0}, \mu^{1}, \mu^{2}, \ldots, \mu^{l}\right)} s_{\mu^{l}},
$$

where $l=u_{1}(\nu)$. Given the code of the partition $\mu^{i-1}, \mu^{i}$ is obtained as follows:

- Starting with the $U$ left of the leftmost $R$ in the code of $\lambda$ and working to the right, move the U's to the right a total of $r_{i}(\nu)$ places by switching a UR to $R U$ in the code $r_{i}(\nu)$ times (so no $U$ can move past the starting point of the next $U$ in the code).

- Let $k(i, j)$ be the number of UR switches made using the last $j$ U's. Then $k(i, 0)=0$, for all $i$.

- $k(i, j) \leq k(i-1, j-1)$, for all $i, j \geq 0$.

Note that this proposition implies that $c_{\mu, \nu}^{\lambda}$ is equal to the number of sequences $\left(\mu=\mu^{0}, \mu^{1}, \mu^{2}, \ldots, \mu^{l}=\lambda\right)$.

Proof: This theorem just follows the computational way to calculate Littlewood-Richardson coefficients, with the only difference being that we use different notation. The sequences $\left(\mu=\mu^{0}, \mu^{1}, \mu^{2}, \ldots, \mu^{l}=\lambda\right)$ are in $1-1$ correspondence to the semistandard Young tableaux of shape $\lambda / \mu$ with 1 's in the boxes in $\mu^{1} / \mu^{0}, 2$ 's in the boxes in $\mu^{2} / \mu^{1}, \ldots$, and $i$ 's in the boxes in $\mu^{i} / \mu^{i-1}$ for all $1 \leq i \leq l=u_{1}(\nu)=l(\nu)$. The restriction that no $\mathrm{U}$ can move past the next $\mathrm{U}$ means that for all $i, \mu^{i} / \mu^{i-1}$ is a horizontal $n$-strip, so the corresponding Young tableau is indeed semistandard. The number of $i$ boxes 
is the number of boxes added to get from $\mu^{i-1}$ to $\mu^{i}$, which is equal to the total number of UR to RU switches made in this step, which is $r_{i}(\nu)$. This means that the Young tableau obtained has shape $\lambda / \mu$ and weight $\nu$. The requirement $k(i, j) \leq k(i-1, j-1)$ means that the number of $i$ 's in the first $j$ rows have is less than the number of $(i-1)$ 's in the first $(j-1)$ rows for all $i$ and $j$. This is equivalent to saying that the reverse-row word is a lattice permutation.

To better illustrate the correspondence between sequences of partitions of the form $\left(\mu=\mu^{0}, \mu^{1}, \mu^{2}, \ldots, \mu^{l}=\lambda\right)$ with the preceding conditions and semistandard Young tableaux, we give the following example.

Example 5.2. Consider the following: $\lambda=(4,3,2), \mu=(2,1)$, and $\nu=$ $(3,2,1)$, and the sequence $\mu^{0}=\mu=(2,1), \mu^{1}=(4,1,1), \mu^{2}=(4,3,1)$, and $\mu^{3}=\lambda=(4,3,2)$. It is straightforward though tedious to verify that this sequence does satisfies the above conditions and hence contributes to $c_{\mu, \nu}^{\lambda}$. If we follow the algorithm in the proof of the theorem and put $i$ 's in each box in $\mu^{i} / \mu^{i-1}$, we get the following semistandard Young tableau:

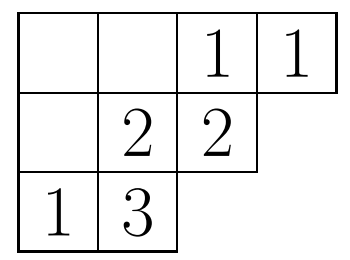

We can also understand this using only the codes of these partitions. Using the algorithm for finding such a sequence, we would find the codes of these partitions (not the partitions themselves) and have the following sequence (omitting leading U's and trailing R's): $\mu^{0}=\mu=\mathrm{RURU}, \mu^{1}=\mathrm{RUURRRU}$, $\mu^{2}=$ RURRURU, $\mu^{3}=\lambda=$ RRURURU. To get from the code of $\mu^{0}$ to the code of $\mu^{1}$ the rightmost $U$ has to move past two $R$ 's (since the number of $R$ 's between this $U$ and the next rightmost $U$ increases by two). This means that we have to add two boxes to the first row of $\mu$ in the first step, which are represented in the semistandard Young tableau with 1's. Similarly, the second $U$ from the right does not have to move past any R's, so there are no boxes added to the second row in the first step thus there are no 1's in the second row in the tableau. Again, the third $U$ from the right must move past one $\mathrm{R}$, so one box is added in the third row and is represented by a 1 in the 
third row of the tableau. Repeating this same proceedure to get from $\mu^{1}$ to $\mu^{2}$ gives us the boxes added in the second step which are represented by 2's in the tableau. Continuing in this way we can find the same semistandard Young tableau using only the codes of the partitions.

Using the codes of partitions we can realize the Pieri Rules in a new way.

Corollary 5.3. (The Pieri Rules)

1. If $\nu=(n)$, then

$$
s_{\mu} s_{\nu}=\sum_{\lambda} c_{\mu, \nu}^{\lambda} s_{\lambda}=\sum_{\lambda} s_{\lambda},
$$

where the sum is over all $\lambda$ such that $\lambda / \mu$ is a horizontal $n$-strip.

2. If $\nu=\left(1^{n}\right)=(1,1, \ldots, 1)$, then

$$
s_{\mu} s_{\nu}=\sum_{\lambda} c_{\mu, \nu}^{\lambda} s_{\lambda}=\sum_{\lambda} s_{\lambda}
$$

where the sum is over all $\lambda$ such that $\lambda / \mu$ is a vertical n-strip.

Proof: For part (1), $s_{\mu} s_{\nu}=\sum_{\left(\mu=\mu^{0}, \mu^{1}=\lambda\right)} s_{\lambda}$, where $\lambda$ is obtained from the code of $\mu$ by moving the U's to the right a total of $n$ places, with no $\mathrm{U}$ moving past the starting point of the next $\mathrm{U}$ in the code. Thus for any $\mathrm{R}$ in the code of $\mu$, at most one $\mathrm{U}$ is moved past this $\mathrm{R}$. But since the number of U's moved past the $i^{\text {th }} \mathrm{R}$ from the left is the number of boxes added to the $i^{\text {th }}$ column, this implies that no two of the added boxes are above each other, so $\lambda / \mu$ is a horizontal $n$-strip. Since $l=u_{1}(\nu)=1$, each sequence has length 2 , so the third condition in the theorem, $k(i, j) \leq k(i-1, j-1)$, is satisfied trivially. For each $\lambda$, the multiplicity of $s_{\lambda}$ in the summation is the number of sequences $\left(\mu=\mu^{0}, \mu^{1}=\lambda\right)$ which is one. Therefore $c_{\mu, \nu}^{\lambda}=1$ if $\lambda$ is a horizontal $n$-strip, and $c_{\mu, \nu}^{\lambda}=0$ otherwise.

For part (2), $s_{\mu} s_{\nu}=\sum_{\left(\mu=\mu^{0}, \mu^{1}, \ldots, \mu^{n}=\lambda\right)} s_{\lambda}$, where $\mu^{i}$ is obtained from the code of $\mu^{i-1}$ by switching one UR to RU, and $k(i, j) \leq k(i-1, j-1)$, for all $i, j \geq 0$. This restriction on $k(i, j)$ implies that the $\mathrm{U}$ moved to get from $\mu^{i}$ to $\mu^{i+1}$ is left of the $\mathrm{U}$ moved to get from $\mu^{i-1}$ to $\mu^{i}$. But since the number of $\mathrm{R}$ 's moved past the $i^{\text {th }} \mathrm{U}$ from the right is the number of boxes added to the $i^{\text {th }}$ row of $\mu$, this implies that no two of the added boxes are in the same row, so $\lambda / \mu$ is a vertical $n$-strip. For each $\lambda$ such that $\lambda / \mu$ is a vertical $n$-strip, the 
only way for the sequence $\left(\mu=\mu^{0}, \mu^{1}, \ldots, \mu^{n}=\lambda\right)$ to end with the partition $\lambda$ is for the rightmost box in $\lambda / \mu$ to be added first, then the next furthest right, and so on. Since there is only one way to do this, the multiplicity of $s_{\lambda}$ in the summation is one. Therefore $c_{\mu, \nu}^{\lambda}=1$ if $\lambda$ is a vertical $n$-strip, and $c_{\mu, \nu}^{\lambda}=0$ otherwise.

\section{References}

[CG] S. R. Carrell and I. P. Goulden. Symmetric functions, codes of partitions and the KP hierarchy, arXiv:0902.4441

[DJKMO] E. Date, M. Jimbo, A. Kuniba, T. Miwa, M. Okado, A new realization of the basic representation of $A_{n}^{(1)}$, Lett. Math. Phys. 17 (1989), no. 1, 51-54.

[J1] N. Jing, Vertex operators, symmetric functions, and the spin Group $\Gamma_{n}$, J. Algebra 138 (1991), no. 2, 340-398.

[J2] N. Jing, Symmetric polynomials and $U_{q}\left(\widehat{s l_{2}}\right)$, Rep. Theory 4 (2000), 46-63.

[M] I. G. Macdonald, Symmetric functions and Hall polynomials, Oxford, 1998.

[O] A. Okounkov, Infinite wedge and random partitions, Selecta Math. (N.S.) 7 (2001), no. 1, 57-81.

[Sa] B. E. Sagan, The symmetric group: representations, combinatorial algorithms, and symmetric functions, Springer, New York, 1991.

[Sch] I. Schur, Über die Darstellung der symmetrischen und der alternierenden Gruppe durch gebrochene lineare Substitutionen, J. Reine Angew. Math. 139 (1911), 155-250.

[St] R. P. Stanley, Enumerative combinatorics, Vol. 2. Cambridge University Press, 1999.

[Ste] J. R. Stembridge. Shifted tableaux and the projective representations of symmetric groups, Adv. Math. 74 (1989), no. 1, 87-134. 
[Z] A. Zelevinsky, Representations of finite classical groups, A Hopf algebra approach, LNM 869, New York, 1981. 